\title{
Orbital Dermoid Cyst With Intratumoral Inflammatory Hemorrhage
}

\author{
-Case Report-
}

\author{
Kentaro KUDO, Satoshi TsuTsumi, Yasuo SUGA, Hidehiro OKURA, \\ Yusuke ABE, Yukimasa YASUMOTO, and Masanori ITO
}

Department of Neurological Surgery, Juntendo University Urayasu Hospital, Urayasu, Chiba

\begin{abstract}
An 85-year-old female presented with a 3-month history of worsening hyperlacrimation and blurred vision following blunt head injury. Her past medical history was unremarkable. Her blood examination identified no abnormal findings. Neuroophthalmological inspection revealed extraocular movement disorder and mild exophthalmos on the affected side. Her visual acuity was not disturbed. Neuroimaging showed a cystic mass located extraconally in the superolateral compartment of the anterior orbit, without enhancement by contrast medium. Surgical exploration resulted in escape of chocolate-colored, liquefied hematoma during dissection. No vascular lesion was found. A grayish-white cyst wall was found adhering strongly to the lateral and superior rectus muscles. The lacrimal gland was not identified. The mass was totally resected. Histological examination indicated dermoid cyst with inflammatory interstitial hemorrhage. No component of vascular malformation or atypia was found. Hyperlacrimation and extraocular movement improved postoperatively. We thought that the previous blunt head injury might have induced intratumoral hemorrhage in the preexisting dermoid cyst and that the escaped contents caused inflammatory irritation, resulting in the hyperlacrimation. Dermoid cyst may show atypical presentation with intratumoral hemorrhage and should always be included in the differential diagnosis of orbital tumor.
\end{abstract}

Key words: dermoid cyst, intratumoral hemorrhage, traumatic episode, orbital tumor

\section{Introduction}

Mass lesions arising from the lacrimal gland and fossa occur as subcutaneous lesions in the superolateral compartment of the anterior orbit and are estimated to account for about $5-13 \%$ of orbital masses. ${ }^{5,10)}$ About half of these diverse pathological entities are thought to be tumors of epithelial origin and the others originate in lymphoid or inflammatory diseases. Pseudotumor, metastasis, and developmental cysts are unusual in the lacrimal fossa. ${ }^{1,5)}$ We present a case of orbital dermoid cyst with a manifestation of intratumoral inflammatory hemorrhage, which might have been caused by previous blunt head injury.

\section{Case Report}

An 85-year-old female consulted a local ophthal-

Received October 18, 2007; Accepted April 14, 2008 mologist complaining of hyperlacrimation and blurred vision in the left eye that had worsened for 3 months. She was referred to our department with suspected orbital tumor. Her past medical history was unremarkable for hypertension, hematological disorder, malignant disease, thyroid disease, sarcoidosis, and paranasal sinus surgery, but she had undergone uneventful cataract surgery of the affected eye 5 years before, and had suffered a blunt injury to the forehead 1 year before.

On admission, examination found a painless, palpable subcutaneous mass in the superolateral compartment of the left orbit with mild exophthalmos and inferolateral ocular displacement. Conjunctival chemosis was not present and the skin overlying the mass was intact without periorbital ecchymosis. Neuroophthalmological inspection revealed no disturbance of visual acuity. Extraocular movements were highly restricted on both lateral and superior gaze to the ipsilateral side without pain, but the light reflex was preserved and isocoric. She was afebrile, and blood examination showed white blood cell 


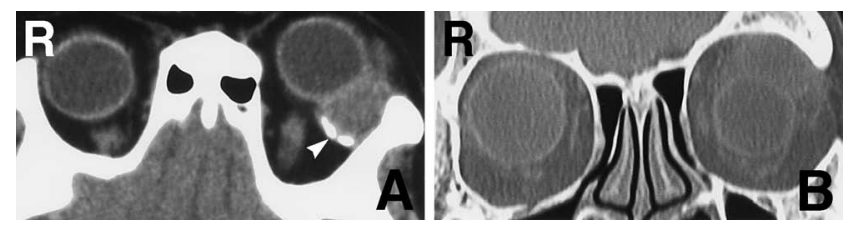

Fig. 1 A: Axial computed tomography scan showing a cystic mass located in the superolateral compartment of the left anterior orbit, with marginal calcification in the inferomedial part of the wall (arrowhead). B: Coronal bone-targeted computed tomography scan demonstrating no bony erosions around the lacrimal fossa.
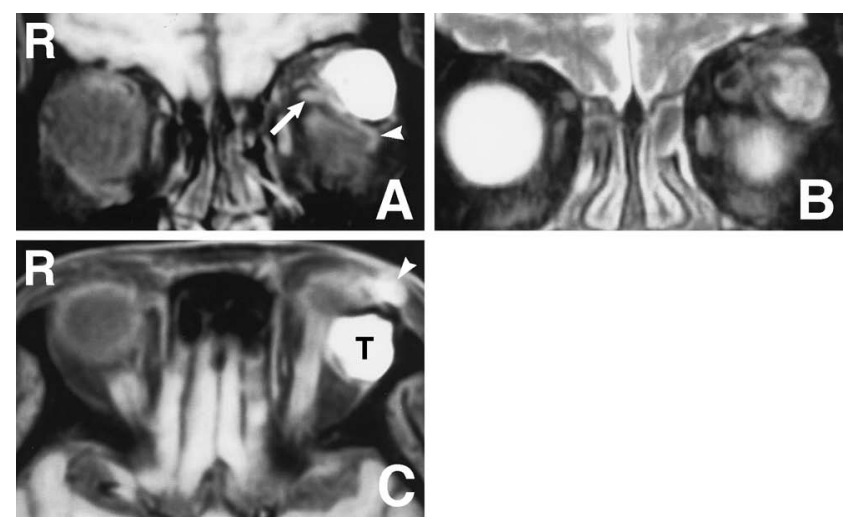

Fig. 2 A, B: Coronal $T_{1^{-}}(A)$ and $T_{2}$-weighted (B) magnetic resonance (MR) images showing the mass as hyperintense and heterogeneously intense, respectively, separate from the superior (arrow) and lateral rectus muscles (arrowhead) without detectable lacrimal gland. C: Axial $T_{1}$-weighted $M R$ image with gadolinium infusion revealing an enhanced mass suggestive of the lacrimal gland (arrowhead) without abnormal vasculature. T: tumor.

count of $5500 / \mu \mathrm{l}$ and C-reactive protein of less than $0.3 \mathrm{mg} / \mathrm{dl}$, in addition to no indications of coagulopathy, endocrinopathy, or underlying malignancy.

Computed tomography (CT) revealed a cystic mass of $2.5 \mathrm{~cm}$ maximal diameter located in the superolateral compartment of the anterior orbit of the affected side, separate from the optic nerve. The cyst contents appeared as slightly low density with marginal calcification in the inferomedial part of the isodense wall. The orbital bony structures were intact (Fig. 1). Magnetic resonance imaging showed the mass as hyperintense on $\mathrm{T}_{1}$-weighted images and heterogeneously intense on $\mathrm{T}_{2}$-weighted images,

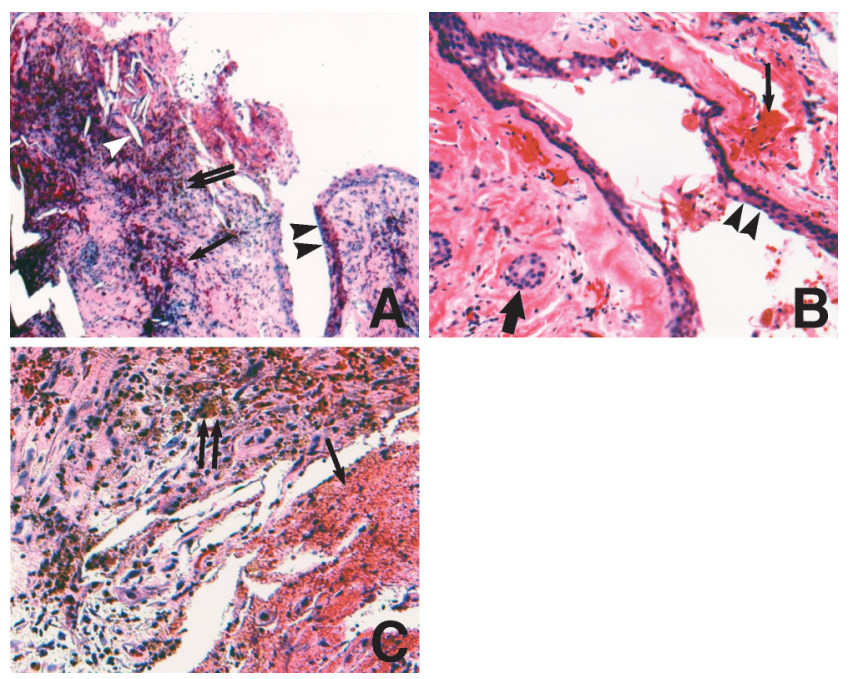

Fig. 3 Photomicrographs of the cyst wall showing squamous epithelial lining (A, B; double arrowheads) compatible with dermoid cyst with interstitial hemorrhagic change (A-C; arrow), cholesterin clefts (A; arrowhead), hemosiderin-laden macrophages $(A, C$; double arrows), dermal appendages (B; thick arrow), and lymphocytic infiltration, without atypia or vascular malformation components. Hematoxylin and eosin stain, original magnification $A: \times 40 ; B, C: \times 250$.

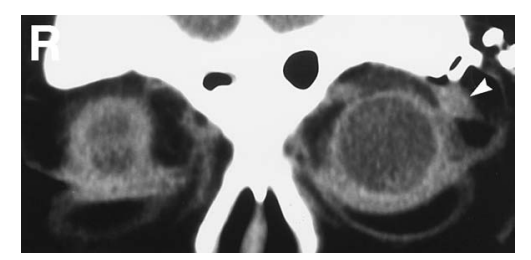

Fig. 4 Postoperative coronal computed tomography scan showing total tumor resection with restoration of the displaced lacrimal gland (arrowhead).

separate from the superior and lateral rectus muscles, without enhancement after gadolinium administration. The optic nerve was distinguished from the lesion. The lacrimal gland was suggested to be located anteriorly separate from the mass, on the affected side. No vascular malformation was found (Fig. 2).

Surgery was performed on May 7, 2007. A linear skin incision of $4 \mathrm{~cm}$ length was made posterior to the lateral canthus parallel to the lateral orbital rim followed by osteoplastic lateral orbitotomy. The lateral orbital wall and periorbita were found intact. Chocolate-colored, pressurized fluid hematoma es- 
caped during the dissection. Grayish-white cyst wall with granulomatous tissue was recognized adhering strongly to the medial surfaces of the lateral and superior rectus muscles. Dissection of the wall was accompanied by continuous bleeding. The lacrimal gland was not identified intraoperatively. The tumor was totally resected en bloc.

Histological examination of the cyst wall revealed squamous epithelial lining with inflammatory granulation tissue, which contained capillary proliferation and interstitial hemorrhage, cholesterin clefts, hemosiderin-laden macrophages, and dermal appendages with lymphocytic infiltration. Those findings were compatible with dermoid cyst with hemorrhagic change. No atypia or vascular malformation component was found (Fig. 3).

The postoperative course was uneventful. Postoperatively, hyperlacrimation resolved without symptoms of hypolacrimation. Extraocular movement in both the lateral and superior directions improved markedly. CT indicated total tumor removal and restoration of the displaced lacrimal gland (Fig. 4).

\section{Discussion}

Developmental cysts found in the orbit and periorbital structures are frequently dermoid cysts, epidermoid cysts, and teratomas. Such lesions arise from inclusion of an ectodermal element during neural tube closure, between 3 and 5 weeks of gestation, by trapping of dermal elements in the suture line, diploe, meninges, or scalp. Dermoid and epidermoid cysts are both true ectodermal inclusion cysts with epithelial lining and may arise at any age in the subcutaneous tissues and along the lines of embryological closure and fusion, most commonly on the face around the eyes because of traumatic or iatrogenic implantation that displaces skin elements from the surface into the underlying tissues. Orbital dermoid cysts are the most common congenital lesions of the orbit, accounting for $1-2 \%$ of orbital masses. These typically unilocular masses are found extraconally in the superior temporal quadrant of the orbit in more than $80 \%$ of cases, most frequently adjacent to the frontozygomatic suture line, with occasional bony erosion. ${ }^{5,9,11,13)}$ The lacrimal gland and fossa masses appear as subcutaneous lesions in the superolateral aspect of the orbit and are estimated to comprise about $5-13 \%$ of the orbital masses. ${ }^{5,10)}$ Diverse pathological entities arise from the lacrimal gland and fossa, about half of them are thought to be tumors of epithelial origin and the rest are lesions that arise from lymphoid or inflammatory diseases. Pseudotumor, metastasis, and developmental cysts may also infrequently occur in the lacrimal fossa. ${ }^{1,5)}$

Dermoid cysts with hemorrhagic change are rare and bled after blunt trauma or spontaneously. ${ }^{2,7,8)}$ Capillary proliferation in and around the dermoid cyst wall, which is susceptible to chemical inflammation, may bleed after subtle mechanical or chemical triggers. Intratumoral hemorrhage can occur in the presence of hypervascularity, neoplastic or abnormal vasculature, hematological disorder, coagulopathy, underlying malignant etiology, and traumatic episode.

In the present case, the dermoid cyst became symptomatic 1 year after blunt injury to the forehead without other contributing factors. The patient had undergone uneventful cataract surgery in the affected eye 5 years before, which was considered unlikely to have implanted the epidermal element into the lacrimal gland or adjacent subcutaneous tissue. Therefore, we assume that preexisting congenital dermoid cyst located posteriorly to the lacrimal gland, as suggested by presurgical neuroimaging, was the origin of the intratumoral hemorrhage after the blunt head injury 1 year before presentation. Resultant growth of the cyst caused by intratumoral hemorrhage finally led to escape of the cyst contents into the surrounding tissue. Intraoperative findings did not identify the lacrimal gland whereas postsurgical neuroimaging revealed restoration of the gland, which may suggest that the tumor was located separate from the lacrimal gland.

The patient's hyperlacrimation, which resolved postoperatively without hypolacrimation, may have been caused by irritable stimulation of the lacrimal gland induced by the escaped cyst contents. Severe adhesion was found between the cyst wall and extraocular muscles, and was thought to indicate coexisting inflammatory reaction. Compression of the lacrimal gland by the dermoid cyst and coexisting inflammatory granulation tissue may have obscured the lacrimal gland during intraoperative inspection, whereas the lacrimal gland appeared on postoperative neuroimaging. Her extraocular movement disorder probably resulted from inflammatory reaction involving the superior and lateral rectus muscles, which caused no pain and was not identified on neuroimaging.

Conjunctival inclusion cyst is a differential diagnosis that can arise as a congenital or acquired lesion, and is associated with ptosis surgery, lid-lowering surgery, intraorbital surgery, trauma, and strabismus surgery. These cysts are thought to result from the implantation of conjunctival epithelial cells into aberrant locations or from obstruction of the gland of Krause. Primary conjunctival cyst of the or- 
bit is most frequently located in the superior nasal quadrant of the orbit, in contrast to dermoid cyst. Histological examination shows stratified epithelial lining including occasional goblet cells without dermal appendages, which are significant differences from dermoid cyst. ${ }^{3,4,12)}$ Marginal calcification, which is one of the characteristics of orbital dermoid cyst, is rare in conjunctival inclusion cyst. ${ }^{12)}$

Dermoid cyst has diverse appearances on neuroimaging because of the complex contents including apocrine glands, sweat glands, sebaceous glands, and hair follicles, in addition to the inflammatory reactions induced by rupture into the surrounding tissue. Such variable findings may confuse pretreatment diagnosis and hinder discrimination from malignant lacrimal gland tumor.$^{6)}$ Dermoid cyst may show atypical presentation with intratumoral hemorrhage, and should always be included in the differential diagnosis of orbital tumor. Dermoid cyst is curable by surgical intervention.

\section{References}

1) Balchunas WR, Quencer RM, Byrne SF: Lacrimal gland and fossa masses: evaluation by computed tomography and A-mode echography. Radiology 149: 751-758, 1983

2) Chen JC, Chen Y, Lin SM, Tseng SH: Sylvian fissure dermoid cyst with intratumoral hemorrhage: case report. Clin Neurol Neurosurg 108: 63-66, 2005

3) Hussin HM, Majid M, Spencer N, Harrid RA, Potts MJ: Large inclusion cyst after ptosis surgery. Acta Ophthalmol Scand 85: 121-122, 2007

4) Imaizumi M, Nagata M, Matsumoto CS, Nakatsuka K, Kashima K: Primary conjunctival epithelial cyst of the orbit. Int Ophthalmol 27: 269-271, 2007

5) Jung WS, Ahn KJ, Park MR, Kim JY, Choi JJ, Kim BS,
Hahn ST: The radiological spectrum of orbital pathologies that involve the lacrimal gland and the lacrimal fossa. Korean J Radiol 8: 336-342, 2007

6) Karatza EC, Shields CL, Shields JA, Eagle RC Jr: Calcified orbital cyst simulating a malignant lacrimal gland tumor in an adult. Ophthal Plast Reconstr Surg 20: 397-399, 2004

7) Levine RL, Pepe PE, Blackstone W, Danzinger J, Varon J: Occult traumatic avulsion of an ovarian dermoid cyst. Am J Emerg Med 10: 344-346, 1992

8) Mamata H, Matsumae M, Yanagimachi N, Matsuyama S, Takamiya Y, Tsugane R: Parasellar dermoid tumor with intra-tumoral hemorrhage. Eur Radiol 8: 1594-1597, 1998

9) Nugent RA, Lapointe JS, Rootman J, Robertson WD, Graeb DA: Orbital dermoids: features on CT. Radiology 165: 475-478, 1987

10) Shields CL, Shields JA, Eagle RC, Rathmell JP: Clinicopathologic review of 142 cases of lacrimal gland lesions. Ophthalmology 96: 431-435, 1989

11) Smirniotopoulos JG, Chiechi MV: Teratomas, dermoids, and epidermoids of the head and neck. Radiographics 15: 1437-1455, 1995

12) Song JJ, Finger PT, Kurli M, Wisnicki HJ, Iacob CE: Giant secondary conjunctival inclusion cysts: a late complication of strabismus surgery. Ophthalmology 113: 1049.e1-2, 2006

13) Yilmaz T, Unal OF, Altinok G: Pathology quiz case 2. Epidermal inclusion cyst. Arch Otolaryngol Head Neck Surg 127: 1391, 1393, 2001

Address reprint requests to: Satoshi Tsutsumi, M.D., Department of Neurological Surgery, Juntendo University Urayasu Hospital, 2-1-1 Tomioka, Urayasu, Chiba 279-0021, Japan.

e-mail: shotaro@juntendo-urayasu.jp 